ical condition found. He died forty-eight hours later. The autopsy was limited to an examination of a specimen of membranes and inclosed cauda. To the right of the conus medullaris and partly covered by bundles of the cauda was a firm yellowish mass. The various bundles of the cauda were bound together by inflammatory matter. There were irregular thickenings of the dura and numerous adhesions. Part of the specimen showed hemorrhages and great cellular infiltration in the dura. About the cauda the pia was thickened and infiltrated with 'small spherical cells. In the most central nerve bundles the fibres: were nearly all completely degenerated. The third and fourth nerve roots were normal.

A. F.

\title{
CHOREA AND THE APPEARANCE OF DENTITION.
}

(Houel, Nouveau Montpellier Medical, March I9 and 26, I 892.) This paper is a rather peculiar one, insomuch as it shows how eager physicians are to attribute chorea to any of the different affections to which childhood is exposed. The author speaks of two patients who suffered from this affection. One of the patients; a young girl, fifteen years of age, was taken with choreic movements involving the entire body, the onset of which could not be attributed to fright or 'any'excessive emotion. She had suffered from metorrhagia and was profoundly anæmic. The second patient was fourteen years old, and was then suffering from her fourth attack. In both patients the nine-year molars were late in their appear. ance on both sides. The superior incisors are likewise delayed in their appearance. Reasoning from these conditions the writer follows out the idea of his colleague Baumel, who in a previous work gave it as his opinion that chorea is a disease predisposed to by anæmia, and the exciting cause in the precipitation of an attack is the irritation of dentition; that is, the anæmia is the predisposing cause and the dentition is the exciting.

Tedenat was very much concerned by the induration of the arteries which both of these cases presented. And without making the artero-sclerosis as the cause of the chorea, he thinks, that this depraved state of nutrition predisposes to it. And he finds some difficulty in making it clear to himself just what rôle the appearance of dentition plays in causing the attacks. But it in all 
the cases where the attack of chorea appeared with the swelling of the gums previous to the outcoming of the teeth, the symptoms ameliorated when the dentition became complete.

Baumel also noted that chorea was most apt to take place at the period in life; that is, between the ages of six and fifteen, when dentition of the two great molars occurs; the first appearing from the sixth to the twelfth year, and the second from the twelfth to the fourteenth.

Tedenat remarked that this period of life was not given over entirely to the development and appearance of the teeth, but an important process of evolution and development was going on in the ovaries, the uterus, the testicles, which was to bring about a remarkable change in these organs incident and previous to their taking on reproductive activity.

Gilis thought that chorea was a neurosis, which depended for its appearance on two great factors: first, a soil prepared by the inheritancy of certain predilections and diathesis; and, second, some trouble in the evolution of the organism, which is the determining cause. In this connection he had noticed that chorea was more apt to occur in young girls at the time of puberty; and without overlooking the influence of dentition at this time, he thought that the utero-ovarian evolution at this time exercised a far greater influence upon the general organism than did the evolution of dentition (Rev. gén. de méd. de chirurg. et d'obstet., April 27, 1892). J. C.

\section{BERI-BERI: ITS CAUSATION AND TREAT- MENT.}

Scott (Practitioner, May, I892). In the above-quoted paper Dr. Scott gives the results of his experience in the study and treatment of beri-beri as he has seen it among. the Chinese coolies. He discusses the subject clinically, and to some extent theoretically, and attempts to point out that disease, looked at from the clinical side, appears. to arise from an altered condition of the blood, associated with excessive acidity, and that the symptoms are caused by this acidity or by the presence of an alkaloid having an action similar to that of muscarine.

The conclusions that he arrives at are shortly stated as follows:

We may attribute beri-beri, consistently with all the available evidence, to a blood change, with symptoms 